\title{
Significance of Nucleation Kinetics in Sn Whisker Formation
}

\author{
E. CHASON, ${ }^{1}$ F. PEI, ${ }^{1,2}$ C.L. BRIANT, ${ }^{1}$ H. KESARI,${ }^{1}$ and A.F. BOWER ${ }^{1}$ \\ 1.-School of Engineering, Brown University, Providence, RI 02912, USA. 2.-e-mail: fei_pei@ \\ brown.edu
}

\begin{abstract}
Sn whiskers are believed to form in response to stress in layers used as protective coatings. However, what makes them form at specific sites on the surface is not known. We have used thermal expansion mismatch to induce stress and observe the resulting whisker formation. Cross-sectional measurements of the region around whiskers show that there are oblique grain boundaries under the whiskers that are not seen in the as-deposited columnar structure. The kinetics also suggest that the whiskering sites may be formed by a nucleation process. Based on these results, we propose a nucleation mechanism in which the boundaries of the surrounding grains migrate due to strain energy differences and create oblique boundaries at which whiskers can form. A simple model is developed to predict the stress-dependence of the nucleation rate.
\end{abstract}

Key words: $\mathrm{Pb}$-free manufacturing, tin whiskers, thermal cycling

\section{INTRODUCTION}

Sn whiskers are a critical reliability issue in current electronics manufacturing. They grow in the form of long filaments from Sn platings that are used as protective coatings on electronic components and printed circuit boards. Numerous system failures (including satellite crashes and nuclear power plant shutdowns) have been attributed to their growth. ${ }^{1}$ The issue has become more important since the advent of $\mathrm{Pb}$-free manufacturing. ${ }^{2} \mathrm{In}$ the past, $\mathrm{Pb}$ alloying of the $\mathrm{Sn}$ coatings was used to effectively suppress the formation of whiskers. ${ }^{3}$

Many studies have been performed to understand the driving forces and mechanisms underlying whisker formation ${ }^{4}$ (or lower aspect-ratio features that we refer to as hillocks). The cause of whisker/ hillock formation is generally accepted to be stress in the Sn layer. ${ }^{5-7}$ Although the stress may have multiple origins, the formation of intermetallic compounds (IMC) at the $\mathrm{Sn}-\mathrm{Cu}$ interface is the most worrisome source, since it continues to develop as long as the metals react to form IMC. Other sources of stress such as mechanical deformation and residual stress from the initial deposition can be

(Received April 30, 2014; accepted August 16, 2014;

published online September 9, 2014) relaxed by whisker growth or other plastic relaxation processes, so they are less likely to lead to the formation of long whiskers that are the most dangerous.

The underlying mechanism behind whisker growth appears to be the diffusion of atoms from the stressed film to the base of the whisker (driven by local or long-range stress gradients). However, this does not explain why whiskers form at specific sites on the surface, i.e., typically only 1 out of $10^{4}-10^{5}$ grains forms a whisker. $\mathrm{Tu}^{8}$ recognized that cracking of the oxide is necessary for whisker growth and proposed that spots of broken oxide on the surface are the places where the whiskers will grow. To test this, Jadhav et al. ${ }^{9}$ removed the oxide at multiple spots on the surface using a focused ion beam (FIB). They found that whiskers did not grow out of the sites where the oxide was removed. Similarly, $\mathrm{Su}$ et al. ${ }^{10}$ showed that whiskers can grow at the holes in a thick patterned oxide but not all of the holes will develop whiskers. These results indicate that a weak or absent oxide is necessary for whisker growth but is not enough to make whiskers grow.

Other results have shown that whisker formation does not require any pre-existing defects at the site where whiskers start to grow, ${ }^{9}$ although defects such as unremoved flux can promote growth. ${ }^{11}$ Additionally, measurements of the IMC layer 
underneath the Sn have shown that the grains where whiskers form do not have a larger concentration of IMC underneath them than other grains. ${ }^{12}$ In fact, after the Sn layer was removed by etching, there were no obvious features in the remaining IMC layer that indicated where the whiskers/hillocks had formed in the layer above.

The results summarized above suggest that the underlying structure of the Sn layer plays an important role in whisker initiation. One factor that has been considered is grain orientation. X-ray diffraction measurements ${ }^{13}$ indicate that the texture of the Sn layer can affect the tendency to form whiskers. Electron backscattering diffraction (EBSD) measurements ${ }^{14}$ of the alignment of grains that form into whiskers/hillocks show that some orientations are preferred, although there are many similar configuration that do not form into whiskers. Synchrotron measurements by Sarobol et al. ${ }^{15}$ suggest that grains with a large elastic strain energy density are most likely to form into whiskers. However, there are many grains with similar orientations that do not deform, so, although the orientation may play a role in whisker formation, it is not the only determinant.

Alternatively, the grain boundary structure may affect whisker formation. The Sn layers typically have a columnar microstructure, with grains that extend throughout the thickness of the film; the corresponding grain boundaries are primarily vertical in character. For the platings that are used in commercial applications, the grain size is typically comparable to the thickness. In comparison, crosssectional measurements of the microstructure directly under whiskers/hillocks show that it is usually different from the rest of the layer. The grain that contains the whisker/hillock does not extend through the thickness of the layer but instead has an oblique grain boundary underneath it (i.e., a boundary with a horizontal component parallel to the layer surface).

An example of a whisker with such a surface grain boundary at its base is shown in Fig. $1 .{ }^{16}$ The presence of the oblique boundary enables the whisker to be pushed out of the surface by the addition of material at its base. ${ }^{6,16-18}$ This provides a sink for the diffusive flux of atoms driven by the stress gradient and allows whisker growth to relieve stress in the layer. Without such sinks for atoms, diffusional flow cannot relieve stress in the layer since the surface oxide prevents diffusion of atoms to the surface. The presence of internal oblique grain boundaries is also proposed to be the reason why $\mathrm{Pb}$-Sn alloys (which have a more equi-axed microstructure) do not form whiskers since these layers develop significantly less stress than pure Sn layers. ${ }^{6,7}$

The origin of oblique grain boundaries at the base of a whisker/hillock is a challenging puzzle since the as-grown layers are columnar and do not appear to have any surface grains after deposition. Vianco and

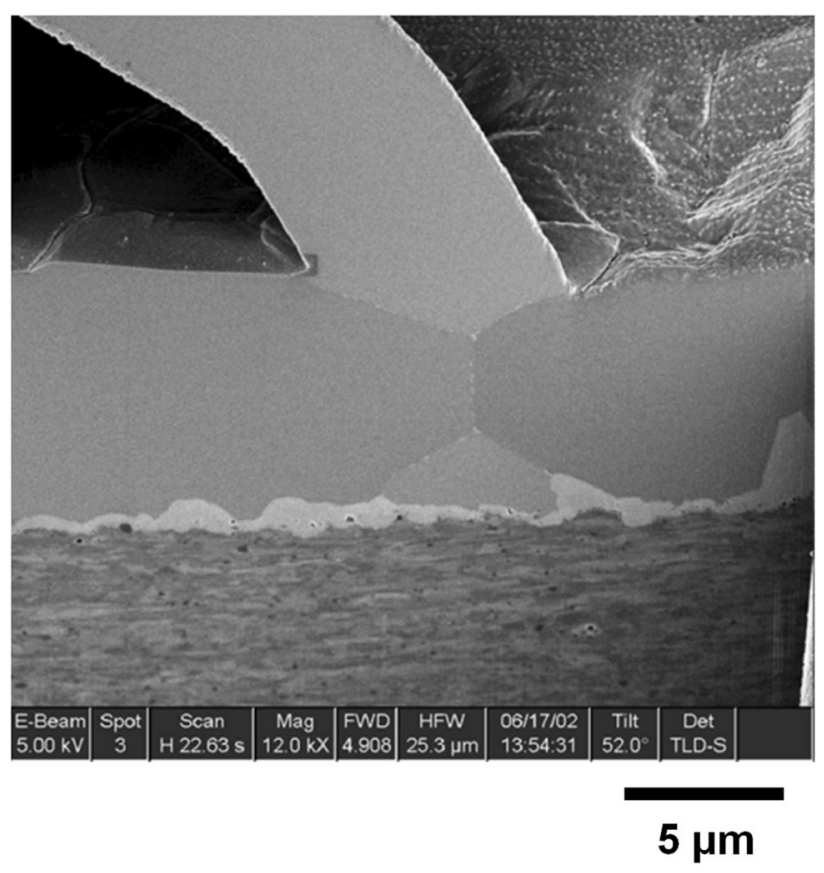

Fig. 1. Cross-section of microstructure at the base of a whisker, showing the presence of an oblique grain boundary (adapted figure reprinted with permission from Ref. 16).

Rejent ${ }^{19}$ have suggested that plastic deformation in the Sn layer creates a driving force for recrystallization. They propose that a new grain nucleates at the surface of a grain that has a high strain energy density. Because the new grain is strain-free, it grows into the deformed grain, producing a nonvertical grain boundary. Subsequently, material flows into the new grain and pushes it out of the layer in the form of a whisker.

To determine if whisker growth corresponds to nucleation of new grains at the surface, Pei et al. ${ }^{14}$ used EBSD to measure the orientation of grains in the Sn layer before any deformation occurred and after they started to form into whiskers/hillocks. They observed grains that did not deform until $40 \mathrm{~h}$ after deposition and found that these grains were already present in the as-grown film, i.e., there was no evidence that they had recrystallized as new grains. Similarly, real-time scanning electron microscope (SEM) videos of whisker and hillock growth ${ }^{9}$ show that surface features appear to grow out of grains that were already there, not from a new grain that nucleated after the layer was grown.

In this work, we propose an alternative mechanism for the formation of surface grains that does not require nucleation of a new grain. We compare this mechanism with the results of experiments using thermal cycling that enable the stress and whisker/hillock nucleation rate to be measured simultaneously. We find that the grain structure underneath the whisker/hillocks is consistent with the microstructure predicted by this mechanism. In addition, the nucleation rate increases with stress 
as predicted by the model. However, the model predicts that the magnitude of the stress needed to nucleate a surface grain is much larger than what we observe. This suggests that the barrier to nucleation in the experiments is lower than the mechanism in the proposed model, or that there are other driving forces for whisker nucleation in addition to the stress.

\section{PROPOSED MECHANISM FOR CREATION OF SURFACE GRAINS IN A COLUMNAR MICROSTRUCTURE}

At first glance, the experimental results described above regarding the sites for whisker initiation appear to be contradictory: whiskers grow out of grains that are present in the as-deposited film, but the original film does not have any surface grains (i.e., the microstructure is columnar). In this case, where do the oblique grain boundaries come from that are found at the base of the whiskers? How is it possible to create a surface grain out of a thicknessspanning columnar grain without nucleating a new grain?

To resolve this requires a mechanism that leads to the formation of oblique boundaries within an existing grain without changing its orientation. We propose that this can occur by the lower section of the grain being consumed by the grains surrounding it. Many studies of bulk materials ${ }^{20-22}$ have shown that, when a material is deformed, grain boundary motion can occur (i.e., strain-induced grain boundary migration) as a result of differences in strain energy density between grains (e.g., due to the density of dislocations or elastic strain energy). The grain boundary between grains with different strain energy density will migrate from the material with lower strain energy density into the material with higher strain energy density, thus lowering the strain energy in the material. Note that we distinguish this process from that of recrystallization in which entirely new grains are formed within the strained material.

To show how this mechanism can relate to whisker nucleation, we consider the evolution of three columnar grains (shown schematically in Fig. 2a) in which the strain-energy density of the grain in the center is higher than that of the two surrounding grains. The boundaries migrate into the grain with higher strain energy to lower the overall strain energy of the material (Fig. 2b). We assume that the grain boundary is pinned at the top surface (by grooves) and at the bottom surface (by intermetallic particles) so that these points cannot move. As the grain boundaries bow in, they eventually meet to form a new segment of vertical grain boundary (Fig. 2c). At the same time, they form a surface grain whose oblique boundaries form a triple point with the newly formed columnar boundary. This could then become the site at which a whisker could grow out of the surface (Fig. 2d). Importantly, the (a)

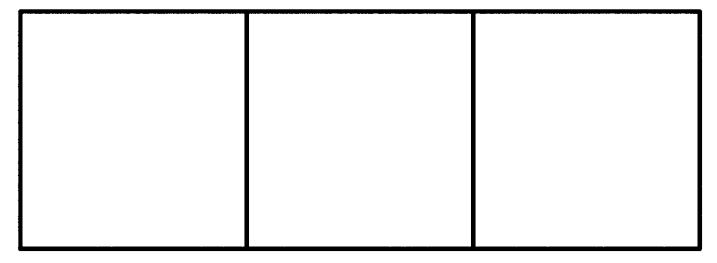

(b)

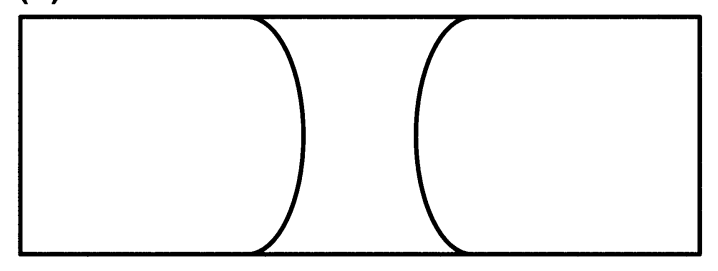

(c)
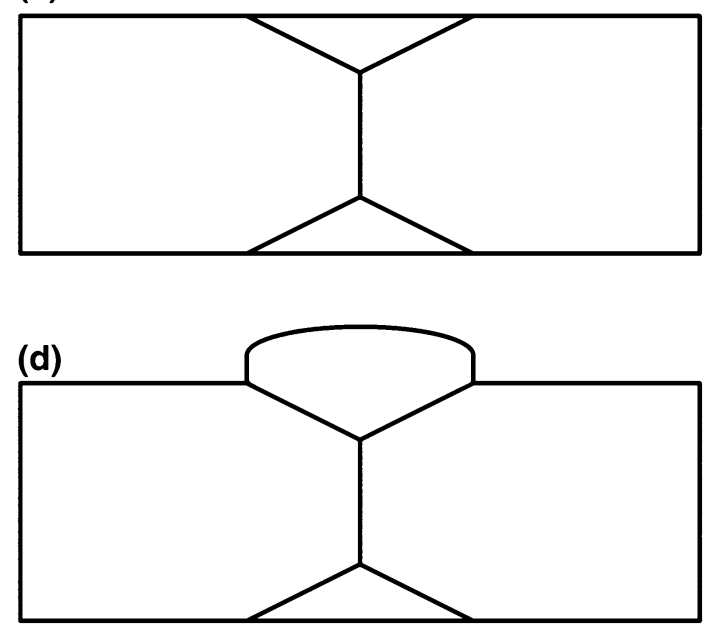

Fig. 2. Schematic describing the proposed mechanism for creation of surface grains by strain-induced grain boundary migration. (a) Initial microstructure consisting of columnar grains. The central grain has larger strain-energy density than its neighbors. (b) The boundaries of the neighboring low-energy grains bow into consume the central grain. (c) The migrating grain boundaries meet to form a new segment of vertical grain boundary, creating an oblique grain boundary and a surface grain. (d) A whisker/hillock feature grows out of the surface grain.

orientation of the surface grain is the same as the orientation of the original grain, consistent with our EBSD observations.

\section{EXPERIMENTS}

To explore the consequences of this mechanism, we have performed measurements of stress and whisker evolution in Sn layers. As discussed above, in most applications the stress is due to the formation of IMC in the layer. The stress in these systems is difficult to characterize and control. To controllably study the dependence of whisker nucleation on stress, we have developed a system in which we use the difference in thermal expansion between the $\mathrm{Sn}$ layer and the Si substrate to create strain when the 

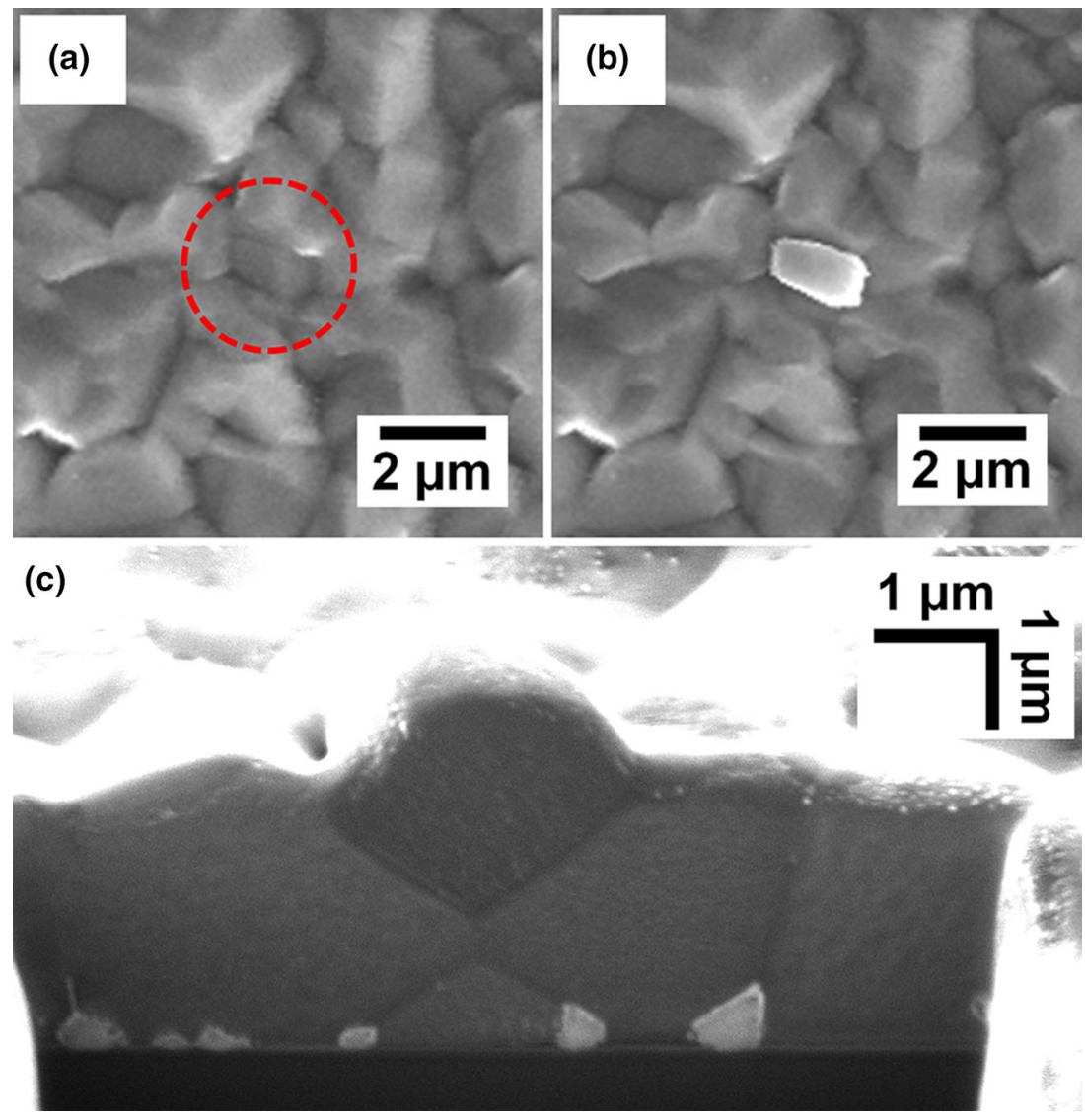

Fig. 3. Measurements of morphology and microstructure of hillocks created by thermally-induced strain. (a) Surface before thermal cycle. (b) Hillock observed growing out of grain in (a) (within the dashed circle) after heating. (c) Cross-section of hillock feature (different from b) showing oblique grain boundary. The scale in the vertical direction in (c) has been corrected for foreshortening due to the angle of incidence.

system is heated (i.e., the resulting strain is proportional to the change in temperature). This enables us to precisely control the applied stress and strain rate. We have implemented this on a measurement system ${ }^{23}$ that enables us to simultaneously monitor the stress (using a wafer curvature apparatus ${ }^{24}$ ) and the whisker density (using an optical microscope).

The samples consist of a $\mathrm{Si}$ substrate with native oxide that has been coated via e-beam evaporation with a $25-\mathrm{nm}$ Ti binding layer and $80-\mathrm{nm} \mathrm{Cu}$ layer. This is then electroplated with $2.5 \mu \mathrm{m}$ of $\mathrm{Sn}$. The thin $\mathrm{Cu}$ layer is used to create the same microstructure and composition (i.e., with a concentration of $\mathrm{Cu}$ in the $\mathrm{Sn}$ that is at the solubility limit) as in other studies of $\mathrm{Sn}$ on $\mathrm{Cu}$ conductors. The sample is held for 9 days after deposition in order to let the Sn react with the $\mathrm{Cu}$ and saturate the formation of IMC. Therefore, at the time when the experiments are done there is no further IMC formation and we can attribute all the stress changes to the thermal strain and relaxation in the film.

Samples were measured during thermal cycles in which the temperature was raised at a constant rate $\left(2^{\circ} \mathrm{C} / \mathrm{min}\right)$ to a final temperature $\left(65^{\circ} \mathrm{C}\right)$ and then held for extended periods $(5 \mathrm{~h})$. Further details of the experiments and the characterization methods can be found in Ref. 23 .

\section{RESULTS}

The morphology and microstructure of typical hillock features induced by the thermal cycle are shown in Fig. 3. The upper section of the figure shows SEM images of the same region of the surface (a) before and (b) after thermal cycling. The similarity in the shape of the grain before heating and the subsequent hillock that grows out of the surface suggests that the hillock grows from a pre-existing grain (this similarity between the hillock shape and the initial grain structure was found for multiple features). The cross-section of a different thermallyinduced hillock is shown in Fig. 3c. The hillock has a V-shaped groove underneath it that connects at its vertex with a vertical boundary between the underlying grains. This microstructure is similar to the microstructure predicted by the strain-induced grain boundary migration model (Fig. $2 \mathrm{~d}$ ) and the IMC-induced whisker shown in Fig. 1.

Measurements of the temperature history and the corresponding layer stress and whisker density are shown in Fig. $4 a-c$, respectively, for a sample 

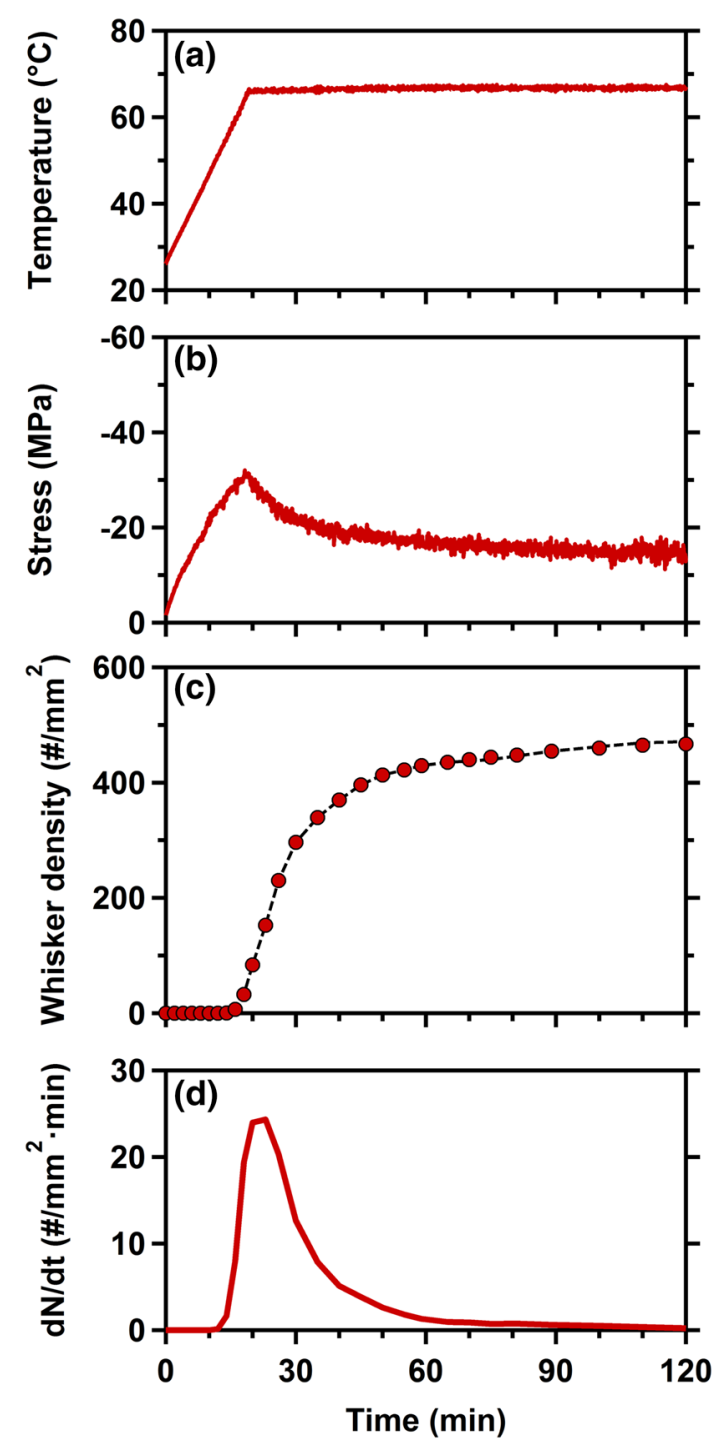

Fig. 4. (a) Temperature, (b) stress and (c) whisker density measured during heating of sample to $65^{\circ} \mathrm{C}$. The axis of stress (in b) is inverted to enable direct comparison with the thermal history (in a). (d) Nucleation rate calculated from whisker density in (c).

heated to a maximum temperature of $65^{\circ} \mathrm{C}$ at a rate of $2^{\circ} \mathrm{C} / \mathrm{min}$. In the early stages, the stress change is observed to be proportional to the temperature change, indicating that there is no stress relaxation in the film. At longer times (higher temperatures), the film stress deviates from proportionality with the temperature indicating the onset of plastic relaxation processes. This change in the stress behavior corresponds to the initiation of whisker nucleation seen in Fig. 4c.

The nucleation rate derived from the measurements of the whisker density is shown in Fig. 4d. The rate increases as the stress is increasing and then decreases as the stress relaxes. Note that the nucleation rate goes to zero when the stress is below a value of $\sim-15 \mathrm{MPa}$. This suggests that there is a critical value below which whiskers cannot grow

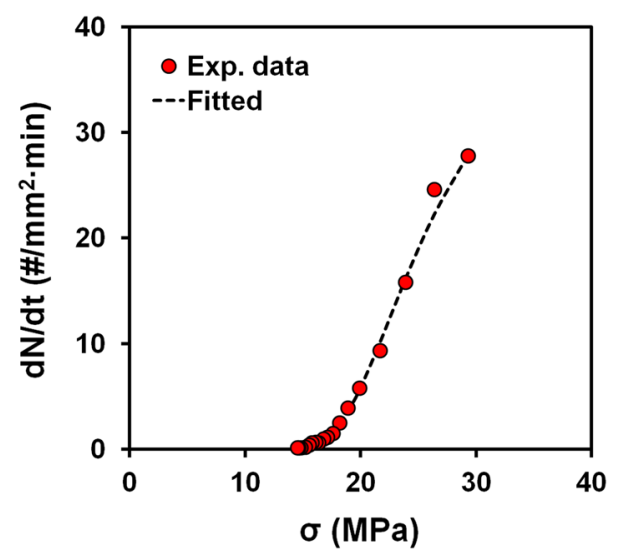

Fig. 5. Dependence of measured nucleation rate on stress from measurements in Fig. 4 (symbols). The dashed line is a fit to the model described in the text.

and is consistent with measurements that show the stress relaxation stops at approximately this value. ${ }^{25}$ The presence of a critical stress for whisker growth has also been suggested in a growth model by Sarobol et al. ${ }^{18}$

Since we have measured the stress and nucleation rate simultaneously, we can also determine the dependence of the nucleation rate on the stress. However, our optical measuring system can only measure the nuclei after they reach a critical size of $\sim 0.5 \mu \mathrm{m}^{3}$. We account for this time lag by using results from another study ${ }^{25}$ which show how the growth rate depends on temperature and stress. We use the growth kinetics to correct the measurements of $\mathrm{d} N / \mathrm{d} t$ for the amount of time that it takes the whisker/hillock to reach an observable volume. This has the effect of shifting the peak in the nucleation rate so that it more nearly coincides with the peak in the stress measurement.

The measurement of $\mathrm{d} N / \mathrm{d} t$ versus stress with this time shift applied is shown in Fig. 5. The dependence on the stress is seen to be fairly strong, changing by a factor of 10 over the range of measured stress. In other work, ${ }^{26}$ we have analyzed these kinetics to determine the dependence of the energetic barrier to nucleation on the applied stress. In the current work, we relate the stress-dependent nucleation kinetics to our proposed mechanism of strain-induced grain boundary migration.

\section{DISCUSSION}

The experimental results have several features that can be compared with the nucleation mechanism described above. The first point is that many of the nuclei seen in the cross-sections have a morphology similar to the cross-section in Fig. 3c. The whisker or hillock is observed to grow from a surface grain whose boundaries form a triple point with a columnar grain (similar to the morphology shown in Fig. 2d). The columnar grain intersects the base of the whisker/hillock in the middle of the surface 
grain, consistent with the idea that the grain boundaries beneath the surface grain came together to form a new boundary. If the grain at the base of the whisker had nucleated at the surface of a single columnar grain (e.g., by recrystallization of a new grain), we might have expected it to have only one grain below it (i.e., not to span two grains with a columnar grain boundary underneath it). We also would not expect it to have the same orientation as the grain before nucleation.

To explore the proposed mechanism further, we can estimate the dependence of the nucleation rate on the strain energy. Micrographs have shown that strain-induced grain boundary motion often occurs by finger-like projections along the grain boundary rather than general motion of the entire boundary. ${ }^{27}$ This suggests that the boundary motion is limited by nucleation of fluctuations in the grain boundary position. In this case, the decrease in strain energy is accompanied by an increase in grain boundary area and two competing terms (strain energy reduction and surface energy increase) must be considered in describing it.

This process can be modeled following the same approach for strain-assisted grain boundary migration that has been proposed for bulk materials. ${ }^{27}$ For the growth of a spherical region of radius $r$, the change in free energy per unit volume, $\Delta G$, is given by

$$
\Delta G=\left(4 \pi r^{2} \Delta \gamma-4 / 3 \pi r^{3} E_{\mathrm{v}}\right) S(\theta),
$$

where $\Delta \gamma$ is the difference in grain boundary energy due to the fluctuation and $E_{\mathrm{v}}$ is the energy difference per unit volume between the material swept out by the migrating boundary and the more highly strained material that it replaces. $\mathbf{S}(\theta)$ is an unspecified shape function that depends on the contact angle and accounts for the fact that the fluctuation may nucleate on an existing boundary and not homogeneously.

Equation 1 can be differentiated with respect to $r$ to determine the critical radius, above which the growth of the strain-relaxed region should proceed with a decrease in the total energy. The expression for this critical radius is given as

$$
r *=2 \Delta \gamma / E_{\mathrm{v}}
$$

If we substitute this expression into Eq. 1 we obtain an expression for the energy barrier for nucleation of a grain boundary fluctuation:

$$
\Delta G^{*}=\left[16 \pi \Delta \gamma^{3} / 3 E_{\mathrm{v}}^{2}\right] S(\theta) .
$$

In order to proceed with this approach, we must estimate the strain energy $E_{\mathrm{v}}$. For simplicity, we consider the case where the in-plane stress in the layer $(\sigma)$ is uniform but the individual grains may have different elastic strain energy densities. This is consistent with studies that suggest that whiskers nucleate from grains with high elastic strain-energy density; ${ }^{15}$ such a difference may arise because the grains have different orientations and the elastic constants in Sn are orientation-dependent. It is also possible that strain energy related to dislocations may also provide a driving force for grain growth (this gives a similar estimate for the order of the strain energy if we consider the work performed by thermally straining the system).

Using the assumption that the energy difference is due to the elastic strain energy, we can approximate $E_{\mathrm{v}}$ as

$$
E_{\mathrm{v}}=1 / 2 K \sigma^{2},
$$

where $K=\left[\left(1 / M_{1}\right)-\left(1 / M_{2}\right)\right]$ and $M_{1}$ and $M_{2}$ are the biaxial moduli of the two grains. Substituting Eq. 4 into 3 we obtain

$$
\Delta G^{*}=S(\theta) 64 \pi \Delta \gamma^{3} /\left(3 K^{2} \sigma^{4}\right)
$$

This predicts a strong dependence on stress $\left(\sigma^{-4}\right)$ for the height of the nucleation barrier. In the context of a nucleation model, this would predict a nucleation rate $(\mathrm{d} N / \mathrm{d} t)$ that is given by

$$
\mathrm{d} N / \mathrm{d} t \sim \beta_{c} \exp \left(-\Delta G^{*} / k T\right)
$$

with $\beta_{\mathrm{c}}$ equal to the rate at which a fluctuation of critical size becomes supercritical.

Then, we can write the term in the exponential as

$$
\Delta G^{*} / k T=\sigma_{\text {char }}^{4} / \sigma^{4}
$$

where

$$
\sigma_{\mathrm{char}}=\left(S(\theta) 64 \pi \Delta \gamma^{3} /\left(3 K^{2} k T\right)\right)^{1 / 4}
$$

This defines the characteristic stress $\left(\sigma_{\text {char }}\right)$ that is needed to make the nucleation process occur.

If we assume that $\beta_{\mathrm{c}}$ is independent of stress, then we can perform a least-squares fit of the data in Fig. 5 to the form in Eqs. 6 and 7. This gives a value for $\sigma_{\text {char }}$ at room temperature that is equal to $24 \mathrm{MPa}$. This model calculation of $\mathrm{dN} / \mathrm{d} t$ is shown as the dashed line in Fig. 5. Note that we can also get a good fit if we assume that $\beta_{\mathrm{c}}$ depends linearly on the stress with $\sigma_{\text {char }}=20 \mathrm{MPa}$.

For comparison, we can estimate the value of $\sigma_{\text {char }}$ from the constants in Eq. 7b. Since the parameters are not well known, we use a range of values for the estimate: $\gamma$ can vary between 1 and $100 \mathrm{~mJ} / \mathrm{m}^{2}, S(\theta)$ can vary between 0.01 and 1 , and $K$ can vary between $2 \times 10^{-11}$ and $2 \times 10^{-12} \mathrm{~Pa}^{-1}$. This provides a range of values that predicts $\sigma_{\text {char }}$ is within $142 \mathrm{MPa}-45 \mathrm{GPa}$ at room temperature.

The value of $\sigma_{\text {char }}$ calculated from materials parameters is clearly much larger than the value extracted from the experimental data. There are several reasons that could explain why there is 
disagreement between the model and the experiment. The first is to recognize that the stress that is measured by the curvature apparatus is the average stress in the layers. The stress around dislocation pile-ups in strained layers covered by a passivation layer $^{28}$ may be significantly larger than the values that we measure. Therefore, the stress at the point where the grain growth occurs may be closer to the calculated values than the stress that we observe. Alternatively, our calculations only consider the applied stress as the driving force for grain growth (in the estimate of $E_{\mathrm{v}}$ ). However, we know that there is a significant driving force for grain growth apart from the stress. In our measurements of the microstructure for different layer thickness, ${ }^{4}$ we find that the grain size is greater for films with greater thickness. This indicates that the grain size increases as the layer gets thicker during deposition and that there is significant driving force for grain growth in the as-deposited film. Therefore, the addition of applied stress at these values may induce grain boundary motion because it is not the only driving force. If this is true, it would suggest that the grains where whiskers originate would also have a tendency towards grain growth without stress, e.g., they are small grains surrounded by larger neighbors. This remains to be explored.

It is also possible that there is another process with lower activation barrier that occurs instead of the mechanism proposed here. For instance, arrays of dislocations in the Sn layers are known to form into sub-grain boundaries, ${ }^{29}$ and these may have a lower barrier for forming surface grains than stressassisted grain boundary migration. This possibility and other potential mechanisms are still being considered.

In summary, recent studies ${ }^{23,26}$ suggest that nucleation of surface sites plays an important role in the formation of Sn whiskers. However, the origin of these nuclei has been difficult to reconcile with several observations. The grains that form into whiskers/hillocks often have oblique grain boundaries, yet the films have columnar microstructures that do not show the presence of surface grains prior to the application of stress. EBSD and SEM measurements also suggest that the whiskers/hillocks grow out of pre-existing grains with no change in their orientation between the time the film is deposited and when the whisker starts to grow, i.e., the grain would appear to be present in the as-deposited layer. We have proposed a mechanism in which subsurface grain boundary motion creates a surface grain from a pre-existing grain, thus preserving its surface orientation. This mechanism is consistent with measurements of whisker formation kinetics and the microstructure observed around the base of whiskers. A model based on strain-assisted grain boundary migration predicts that the nucleation rate increases with the stress. However, the magnitude of stress predicted by this mechanism is larger than the observed stress, suggesting that other effects (high local stresses or other driving forces) play a role in nucleation.

\section{ACKNOWLEDGEMENTS}

The authors gratefully acknowledge the support of the NSF-DMR under contract DMR1206138 and useful contributions from Gordon Barr.

\section{REFERENCES}

1. NASA, Multiple examples of whisker-induced failures are documented on the NASA website. http://nepp.nasa.gov/ whisker/.

2. G.T. Galyon, IEEE Trans. Electron. Packag. Manuf. 28, 1 (2005).

3. S.M. Arnold, Plating 53, 1 (1966).

4. E. Chason, N. Jadhav, F. Pei, E. Buchovecky, and A. Bower, Prog. Surf. Sci. 88, 2 (2013).

5. B.Z. Lee and D.N. Lee, Acta Mater. 46, 10 (1998).

6. W.J. Boettinger, C.E. Johnson, L.A. Bendersky, K.W. Moon, M.E. Williams, and G.R. Stafford, Acta Mater. 53, 19 (2005).

7. E. Chason, N. Jadhav, W.L. Chan, L. Reinbold, and K.S. Kumar, Appl. Phys. Lett. 92, 17 (2008).

8. K.N. Tu, Phys. Rev. B 49, 3 (1994).

9. N. Jadhav, E. Buchovecky, E. Chason, and A. Bower, JOM 62, 7 (2010).

10. C.-H. Su, H. Chen, H.-Y. Lee, and A.T. Wu, Appl. Phys. Lett. 99, 13 (2011)

11. P. Snugovsky, S. Meschter, Z. Bagheri, E. Kosiba, M. Romansky, and J. Kennedy, J. Electron. Mater. 41, 2 (2012).

12. F. Pei, N. Jadhav, and E. Chason, JOM 64, 10 (2012).

13. P. Sarobol, A.E. Pedigo, P. Su, J.E. Blendell, and C.A. Handwerker, IEEE Trans. Electron. Packag. Manuf. 33, 3 (2010).

14. F. Pei, N. Jadhav, and E. Chason, Appl. Phys. Lett. 100, 22 (2012).

15. P. Sarobol, W.-H. Chen, A.E. Pedigo, P. Su, J.E. Blendell, and C.A. Handwerker, J. Mater. Res. 28, 05 (2013).

16. J. Smetana, IEEE Trans. Electron. Packag. Manuf. 30, 1 (2007).

17. N. Jadhav, M. Williams, F. Pei, G. Stafford, and E. Chason, J. Electron. Mater. 42, 2 (2013).

18. P. Sarobol, J.E. Blendell, and C.A. Handwerker, Acta Mater. 61, 6 (2013).

19. P.T. Vianco and J.A. Rejent, J. Electron. Mater. 38, 9 (2009).

20. P.A. Beck and P.R. Sperry, J. Appl. Phys. 21, 2 (1950).

21. S.P. Bellier and R.D. Doherty, Acta Metall. 25, 5 (1977)

22. J.E. Bailey and P.B. Hirsch, Philos. Mag. 5, 53 (1960).

23. F. Pei and E. Chason, J. Electron. Mater. 43, 1 (2014).

24. E. Chason, Thin Solid Films 526, (2012).

25. F. Pei, A. F. Bower, and E. Chason, unpublished (2014).

26. F. Pei, C.L. Briant, H. Kesari, A.F. Bower, and E. Chason, Scripta Mater. (2014). doi:10.1016/j.scriptamat.2014.08.019.

27. F.J. Humphreys and M. Hatherly, Recrystallization and Related Annealing Phenomena (Oxford: Pergamon, 1995), pp. 207-209.

28. L. Nicola, E.V. Giessen, and A. Needleman, J. Mater. Res. 19, 04 (2004).

29. K.S. Kumar, L. Reinbold, A.F. Bower, and E. Chason, J. Mater. Res. 23, 11 (2008). 